\title{
The ovarian budget crisis
}

\section{David F. Albertini}

Published online: 25 March 2010

(C) Springer Science+Business Media, LLC 2010

In times of economic peril, much effort is spent searching for the causes and worrying about the short and long term consequences when they have profound effects on our daily well-being and livelihood. The floundering U.S. economy has had global consequences, not the least of which has been the demise of prominence in technology and science that Americans have benefitted from for many years. The delicate balance between revenue and expenditure that equilibrates the budget has a parallel: The human ovary. In this issue, we see evidence of the ovarian budget crisis that is at the base of the "global" economy we call human reproduction. And not too far behind lie implications for long-term outcomes that will likely change how medicine is practiced in and around the field of human ARTs. In this case, building a new knowledge base upon which safer and more efficient patient care can be realized, and not a cataclysmic stimulus rescue effort, is the root to economic recovery.

Toward this end, it is a chance event in the course of basic science that unanticipated results break the foundation on which the field of reproductive biology is built.

Our lead article this month comes from the Editor's laboratory and reflects one of those welcomed surprises that often results from the diligent efforts of a trainee. Since it was identified by Bob Edwards in the 1960s, oocyte maturation has been a seminal problem; one that set the stage for the initial attempts of in vitro fertilization in humans. What Edwards observed in his classical studies

D. F. Albertini $(\bowtie)$

Department of Molecular \& Integrative Physiology,

University of Kansas Medical Center,

3901 Rainbow Boulevard,

Kansas, KS 66160, USA

e-mail: dalbertini@kumc.edu (carried out during a sabbatical in the Jones laboratory at Johns Hopkins) was that human oocytes undergo a remarkable "dance of the chromosomes" over a matter of hours that results in the reduction of chromosome number. This is a prerequisite for the completion of meiosis at the time of fertilization. The choreography that he witnessed began with the oocyte resuming meiosis after a long period of dormancy in the follicle and progressed over a span of hours to the metaphase II stage of meiosis. He recognized this process as "nuclear" maturation to denote where the currency of the female would arrive upon ovulation. Soon thereafter, he and others came to realize that nuclear maturation was simply not all that was required for successful fertilization and the development of the conceptus. This process is what we now call "cytoplasmic" maturation, an array of molecular and cellular alterations that confer upon the oocyte the ability to be fertilized and develop through the preimplantation stages of development. While we now know that the timely coordination of both nuclear and cytoplasmic maturation are at the heart of attaining oocytes of high developmental quality, whether the processes involved common or discrete signaling mechanisms has remained a fundamental mystery in the field of oocyte biology: Enter the findings of this paper.

The driver for nuclear maturation turns out to be a highly conserved protein kinase pathway variously referred to as MPF, H1 kinase, or today, the cyclin/CDK1 class of protein phosphorylating enzymes. Once activated, these kinases orchestrate a series of protein phosphorylations that assure the timely formation, alignment, separation, and posturing of chromosomes in preparation for making mother's genomic contribution to the world population. Interestingly, the biochemical machinery behind nuclear maturation is not what distinguishes an oocyte from any other garden-variety somatic cell. Instead, it is what is happening well outside 
the wonderful world of the genome that carries the burden of our species' destiny. Herein lies the oocyte cortex: An uncharted territory until recently, this domain of the plasma membrane (and an associated cytoskeleton) seems to be modified in such a way as to anchor and store many of the factors that will be called upon to drive early development. What this new work reveals is that a distinct set of signaling molecules that belong to the clan of src family kinases are involved in ensuring that the cortex of the oocyte matures to set the stage for fertilization and development. This finding raises many interesting questions, especially with respect to current approaches to in vitro oocyte maturation and oocyte cryopreservation. Hopefully a new way of viewing this - the final act in the play we call oogenesiswill emerge as we balance the budget of the ovarian reserve.

Just how much of a role our genes play in designing the perfect oocyte remains a subject of much attention. Likewise, the question of why oocytes differ between patients presumably has a basis somewhere among the base pairs encoded in our genomes. The Kasarie laboratory adds an interesting and perplexing note to the genotype phenotype quandary that has been puzzled over for a long time. They report on a penetrating phenotype in a patient characterized by solitary vacuoles that neither displace nor interfere with the formation of the meiotic spindle. While Van Blerkom and others have for years noted the sporadic occurrence of oocyte vacuoles - especially after cryopreservation, these findings illustrate that in some patients, infertility can be attributed to an imbalance in the economy of membranes during the course of oogenesis. Proof that this delicate balance in oocyte membranes is disrupted during cryopreservation is also evidenced by the work of
Coticchio and colleagues, who identify recurrent alterations in the oocyte cortex as a result of the use of slow freeze technology. It will be important to determine if such alterations also occur during vitrification as the debate continues over the best way to cryopreserve human oocytes.

The age-old question of what is it that awakens the chronically sleepy primordial follicle that was put to bed some many years earlier has taken a surprising twist with the recent discovery that these follicles may not be under the influence of an intraovarian sleeping pill after all. Especially in the field of fertility preservation, where it is known that isolated pieces of ovarian cortex cause uncontrolled activation of the primordial follicle reserve, the impetus to define the mechanisms of activation has grown recently. In this issue, some insight into the problem is provided by work from the Telfer laboratory. Based on their studies with human ovarian culture preparations, it now appears that activins may be part of the system that awakens primordial follicles.

And finally, we invite our readership to take full advantage of the scientific underpinnings of premature luteinization in a review by Elnaskor, and the recent liability issues that are raised in the paper by Borakay.

We will continue to bring leading-edge studies in the realm of technological innovations to the pages of JARG. In this issue in particular, we direct your attention to the paper from Nakagawa et al, which describes new devices for embryo cryopreservation and the studies reported by Peleg and her colleagues, which provides a critical assessment of the use of single-cell molecular diagnostics that have been so quickly adopted in the field of preimplantation genetic diagnosis. 